OPEN

SUBJECT AREAS:

NATURAL PRODUCTS

MASS SPECTROMETRY

BIOANALYTICAL CHEMISTRY

BIOCHEMICAL ASSAYS

Received

17 April 2013

Accepted

11 June 2013

Published

1 July 2013

Correspondence and requests for materials should be addressed to

Z.P.Y. (zhongping. yao@polyu.edu.hk)or

W.C.Y. (chywc@

yahoo.com.cn)

* These authors contributed equally to this work.

\section{In Vivo and Real-time Monitoring of Secondary Metabolites of Living Organisms by Mass Spectrometry}

\author{
Bin $\mathrm{Hu}^{1,2 *}$, Lei Wang ${ }^{1,2,3 *}$, Wen-Cai Ye \& Zhong-Ping Yao ${ }^{1,2}$
}

\begin{abstract}
'State Key Laboratory of Chirosciences, Food Safety and Technology Research Centre and Department of Applied Biology and Chemical Technology, The Hong Kong Polytechnic University, Hung Hom, Kowloon, Hong Kong S. A. R., China, ${ }^{2}$ State Key Laboratory of Chinese Medicine and Molecular Pharmacology (Incubation), Shenzhen Research Institute of The Hong Kong Polytechnic University, Shenzhen 518057, China, ${ }^{3}$ Institute of Traditional Chinese Medicine and Natural Products, College of Pharmacy, and Guangdong Province Key Laboratory of Pharmacodynamic Constituents of TCM and New Drugs Research, Jinan University, Guangzhou 510632, China.
\end{abstract}

Secondary metabolites are compounds that are important for the survival and propagation of animals and plants. Our current understanding on the roles and secretion mechanism of secondary metabolites is limited by the existing techniques that typically cannot provide transient and dynamic information about the metabolic processes. In this manuscript, by detecting venoms secreted by living scorpion and toad upon attack and variation of alkaloids in living Catharanthus roseus upon stimulation, which represent three different sampling methods for living organisms, we demonstrated that in vivo and real-time monitoring of secondary metabolites released from living animals and plants could be readily achieved by using field-induced direct ionization mass spectrometry.

econdary metabolites play important roles in the survival and propagation of animals and plants. For example, some animals and plants produce chemicals to resist environmental stress, and some secret toxic chemicals for defense purpose upon attack. Understanding these processes at the molecular level is an integral part of our exploration of nature. Conventional approaches for detection of secondary metabolites typically involve homogenization and extraction followed by analysis of extracts using techniques such as chromatography coupled with mass spectrometry ${ }^{1,2}$. These approaches are not only time-consuming and labor-intensive, they are also unable to provide transient and dynamic information about the metabolic processes. Techniques for in vivo and real-time detection of secondary metabolites from biological systems are thus highly desirable.

Mass spectrometry (MS) is a rapid and sensitive tool for analysis of metabolites from biological samples ${ }^{3-5}$. The development of ambient ionization techniques in recent years allows direct analysis of samples with little or no sample preparation ${ }^{6-9}$. Desorption electrospray ionization $(\mathrm{DESI})^{10,11}$, direct analysis in real-time (DART) ${ }^{12}$, and neutral desorption (ND) coupled with extractive electrospray ionization (EESI) ${ }^{13,14}$ enable direct detection of analytes on surfaces of living organisms at atmospheric pressure. Endogenous compounds of plant tissue could be directly analyzed using techniques such as direct ionization (DI) ${ }^{15}$, leaf spray ${ }^{16}$ and tissue spray ${ }^{17}$. In these techniques, tissue sample is cut into $\mathrm{V}$-shape and connected to a high voltage. With addition of some solvents to the tissue sample if necessary, spray ionization is induced from the tip of the plant tissue and a spectrum is obtained. Leaf spray has been attempted to detect chemical response of cabbage leaf upon punching ${ }^{16}$, and DI has also been used for direct analysis of animal tissue ${ }^{15}$. These techniques, however, require connection of samples to a high voltage (typically $3-5 \mathrm{kV}$ ), which can be a strong stimulus to living plants and animals, and thus may have limited application in in vivo stimulation studies of living organisms.

We herein further develop DI-MS for in vivo studies of living organisms. In this study, the high voltage required for inducing electrospray ionization is applied to the MS inlet, and the studied animal or plant sample is grounded and placed in front of the MS inlet. To differentiate from the previous DI technique that requires connection of the sample to a high voltage ${ }^{15}$, the current technique is termed field-induced DI in this study. We demonstrated that in vivo and real-time detection of secondary metabolites released from both living animals and plants upon stimulation could be easily achieved by using feild-induced DI-MS. 


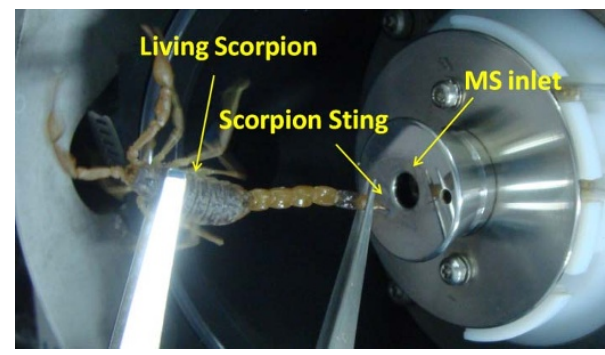

Figure $1 \mid$ Photo of in vivo analysis of a living scorpion by field-induced DI-MS.

\section{Results}

Scorpion uses its sting on the tail as weapon and can release toxic substances from the sting upon attack. Using feild-induced DI, spray ionization can be directly induced from such a sharp sting when the sting is placed close and pointing to the MS inlet, and secretion can be conveniently detected (Fig. 1). In this study, a living scorpion was stimulated at its tail with a pair of tweezers, clear liquid secretion was soon released from the sting, and subsequently spray ionization was induced from the sting and a mass spectrum was obtained (Fig. 2). Compound such as lysine (m/z 147), tryptophan $(\mathrm{m} / \mathrm{z} 205)$ and serotonin $(\mathrm{m} / \mathrm{z}$ 177) could be identified from the spectrum based on their masses and literature searching ${ }^{18-22}$. Serotonin is one of the toxins in scorpion to produce pain ${ }^{21}$, and lysine and tryptophan have been reported to play important roles in the biological activity of the scorpion toxin ${ }^{19}$. Peaks of high abundances were observed in the range of $\mathrm{m} / \mathrm{z} 400-600$. These peaks are singly charged and have a mass interval of $28 \mathrm{Da}$ (e.g., $\mathrm{m} / \mathrm{z} 443.4,471.4,499.5$ and 527.5 , and $\mathrm{m} / \mathrm{z} 487.5$ and 515.4 ) or $16 \mathrm{Da}$ (e.g., $\mathrm{m} / \mathrm{z} 471.4$ and 487.5 , and $\mathrm{m} / \mathrm{z}$ 499.5 and 515.4), and may correspond to lipids according to the literatures ${ }^{23,24}$ and the spectral pattern. When the venom was collected and dissolved in common solvents such as methanol, white precipitate was formed. No significant peaks were observed in the spectra obtained by analysis of the supernatant using conventional ESI-MS.

Toad can release venom from its skin for defense purpose upon attack $^{25}$. In this study, a toad was stimulated at its postauricular gland with a needle (without breaking the skin), and release of white and milky venom was soon observed. A glass capillary containing some methanol was used with one side putting into the secretion and the other side pointing to the MS inlet (Fig. S1). Spray ionization was observed from the capillary tip pointing to the MS inlet and a spectrum was obtained (Fig. 3). Recently, Peng et $a^{26}$ used a capillary for localized analysis of plants. In their method, a quartz capillary was inserted into a plant for sampling and a stainless needle was inserted into the plant to provide a high voltage to induce spray ionization from the capillary. In this study, with a high voltage applied to the MS inlet, spray ionization was conveniently induced from a glass capillary linked to the sample, and no additional power supply device connected to the sample was required. This is particularly important and useful for in vivo study of living animals since connection to a high voltage in such cases is very inconvenient and can cause adverse effects to the animals and severe perturbation to the stimulation studies.

As shown in Fig. 3, compounds including serotonin $\left([\mathrm{M}+\mathrm{H}]^{+}\right.$, $\mathrm{m} / \mathrm{z}$ 177.1), $\mathrm{N}$-methylserotonin $\left([\mathrm{M}+\mathrm{H}]^{+}, \mathrm{m} / \mathrm{z} 191.1\right)$, bufotenin $\left([\mathrm{M}+\mathrm{H}]^{+}, \mathrm{m} / \mathrm{z} 205.1\right)$, bufotenindine $\left([\mathrm{M}+\mathrm{H}]^{+}, \mathrm{m} / \mathrm{z} 219.1\right)$, gamabufotalin $\left([\mathrm{M}+\mathrm{Na}]^{+}, \mathrm{m} / \mathrm{z} 425.2\right)$, bufotalidin $\left([\mathrm{M}+\mathrm{H}]^{+}\right.$, $\mathrm{m} / \mathrm{z} 439.2)$ and cinobufotalin $\left([\mathrm{M}+\mathrm{H}]^{+}, \mathrm{m} / \mathrm{z} 481.2\right)$ could be identified based on masses, MS/MS studies and literature searching (Fig. S3). These compounds were reported as the major compositions of the toad venom, and some of the compounds had been found to have antitumor activity ${ }^{27,28}$. The secretion became solidified and brown after exposure in the air for about $3 \mathrm{~min}$, indicating some chemical reactions occurred to the secretion. No signals were then observed after solidification of the secretion. It was also noticed that a cluster of ions were observed in the $\mathrm{m} / \mathrm{z}$ range of 550-700 Da. Venom compositions with masses corresponding to these peaks had not been reported in previous studies, which were mainly based on analysis of dried toad venoms ${ }^{28}$. Identification of these compounds and studies of their biological functions will be carried out.

Dimeric vinca alkaloids such as vinblastine are powerful anticancer drugs derived from Catharanthus roseus. These drugs are produced within the plant by coupling catharanthine with vindoline. Detailed mechanisms for the assembly are still unclear ${ }^{2}$. These dimeric drugs are found at very low levels in the plant and their commercial productions are very expensive ${ }^{29}$. A recent study by Roepke et $\mathrm{al}^{2}$ revealed that catharanthine and vindoline are spatially separated in the leaves of $C$. roseus. It was hypothesized that when the plant is wounded or attacked, catharanthine and vindoline are brought together to allow the formation of the dimers that can prevent cell division. A living C. roseus was analyzed using the fieldinduced DI technique and the spectrum obtained from a leaf of the plant is shown in Fig. 4a. Catharanthine, vindoline and anhydrovinblastine were predominantly observed in the spectrum. When heating at $450^{\circ} \mathrm{C}$ was induced for $3 \mathrm{sec}$ at $\sim 30 \mathrm{~mm}$ away from the spray tip of the leaf, anhydrovinblastine was found to significantly increase, as shown in Fig. 4b for the full spectrum and Fig. S4a for the change in extracted ion chromatogram for anhydrovinblastine. Repeated measurements with three different leaves revealed that abundance of anhydrovinblastine increased from $25.0 \pm 4.1 \%$ in the spectrum before heating to $93.6 \pm 5.2 \%$ after heating. Increased abundances of potassium adducts were also observed in the spectrum obtained after the heating, along with the appearance of monogalactosyldiacylglycerol (MGDG) (Fig. 4b and Fig. S4b). Accumulation of potassium has been reported as an osmotic adjustment strategy for plants to maintain water ${ }^{30}$, and MGDG was previously reported to accumulate when plants were wounded ${ }^{31}$. Our results confirmed the hypothesis proposed by Roepke et $\mathrm{al}^{2}$, and suggested that production of anhydrovinblastine in $C$. roseus might be increased by changing the living environment of the plant.

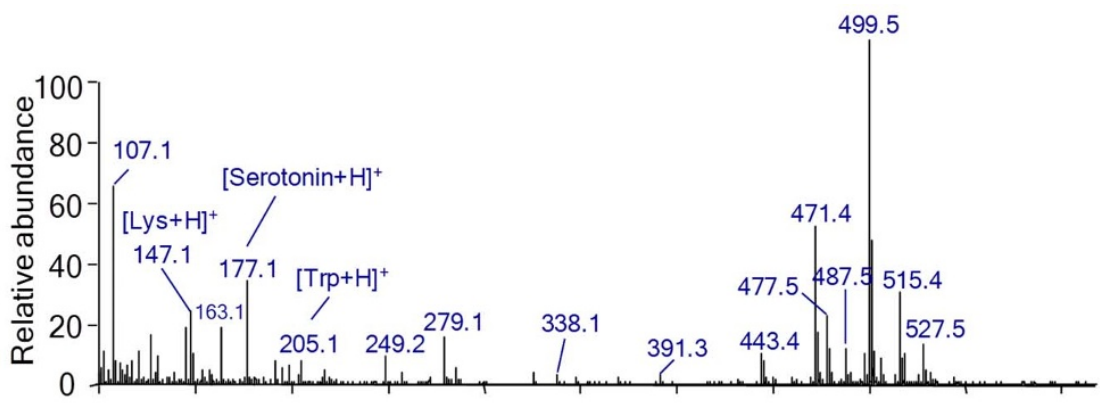

Figure $2 \mid$ Mass spectrum obtained by field-induced DI-MS analysis of the secretion released from a living scorpion upon stimulation. 


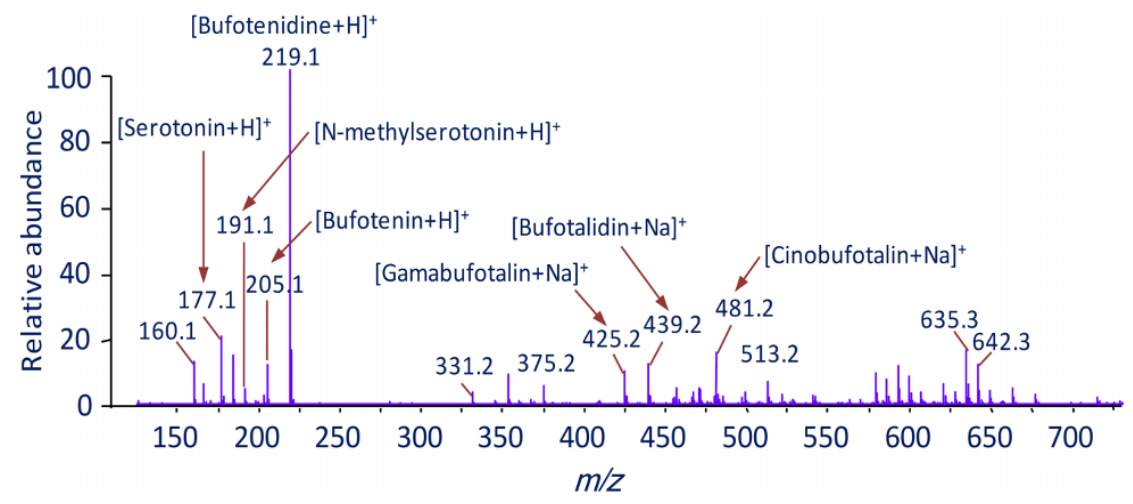

Figure 3 Mass spectrum obtained by field-induced DI-MS analysis of the secretion released from a living toad upon stimulation.

\section{Discussion}

In this study, we presented proof-of-principle for in vivo and realtime monitoring of secondary metabolites released from living organisms using field-induced DI-MS. Without connecting the analyzed samples to a high voltage not only avoids perturbation to the stimulation studies and potential harzard to living organisms, but also greatly facilitates sampling from living organisms. Spray ionization could be conveniently induced from a tip of the studied living organism when the tip is moved close and pointing to the MS inlet. Such a tip can be intrinsic to the living organism (e.g., the sting of a scorpion), or become available by introducing a capillary to the sample (e.g., in the case for in vivo study of toad), or formed by modifying the sample (e.g., cutting the analyzed leaf to a V-shape). We demonstrated that this technique could be readily used for in vivo and real-time monitoring of secondary metabolites released from both living animals and plants upon stimulation. Such in vivo and real-time monitoring is simple, rapid, and able to detect compounds that could not be observed by analysis using conventional approaches. Further development of feild-induced DI-MS, including allowing observation of more compounds of interest in a single spectrum, coupling it to a high resolution tandem mass spectrometer to improve identification of compounds, and applying it for studying other interesting biosystems and clinical studies, are on-going.

\section{Methods}

Materials. Living scorpion (Buthus martensii Karsch; origin: Guanzhou, China), toad (Bufo bufo gargarizans Cantor; origin: Guanzhou, China), and Catharanthus roseus were supplied by College of Pharmacy, Jinan University (Guangzhou, China). Glass capillaries (ID: $1.0 \mathrm{~mm}$, length: $10 \mathrm{~mm}$ ) were purchased from Huaxi Medical University Instrument Factory (Chengdu, China). Methanol was purchased from Tedia (Fairfield, OH, USA). The experiments related to animals were approved by College of Pharmacy, Jinan University.

Feild-induced DI-MS Measurements. Mass spectra were acquired on an Agilent 6210 TOF-MS instrument (Agilent Technologies, Santa Clara, CA, USA) in positive ion mode. The analyzed biological samples were directly placed in front of the MS inlet with a tip pointing to the inlet. Note that when handling living animals such as scorpion and toad, gloves, mask and goggles should be worn for protection purpose. For field-induced DI-MS analysis of scorpion, a living scorpion was held by tweezers and its sting was directly used as the spraying tip (Fig. 1); for field-induced DI-MS analysis of toad, a living toad was held by hand and a glass capillary, which is commonly used for thin layer chromatography (TLC) spotting, was employed as the spraying tip as described in Results and Discussion and shown in Fig. S1; for fieldinduced DI-MS analysis of the potted C. roseus, the analyzed leaf was held by hand and the spraying tip was formed by cutting the leaf into a V-shape (Fig. S2). The distance between the tip and MS inlet was typically $\sim 8 \mathrm{~mm}$. The capillary voltage

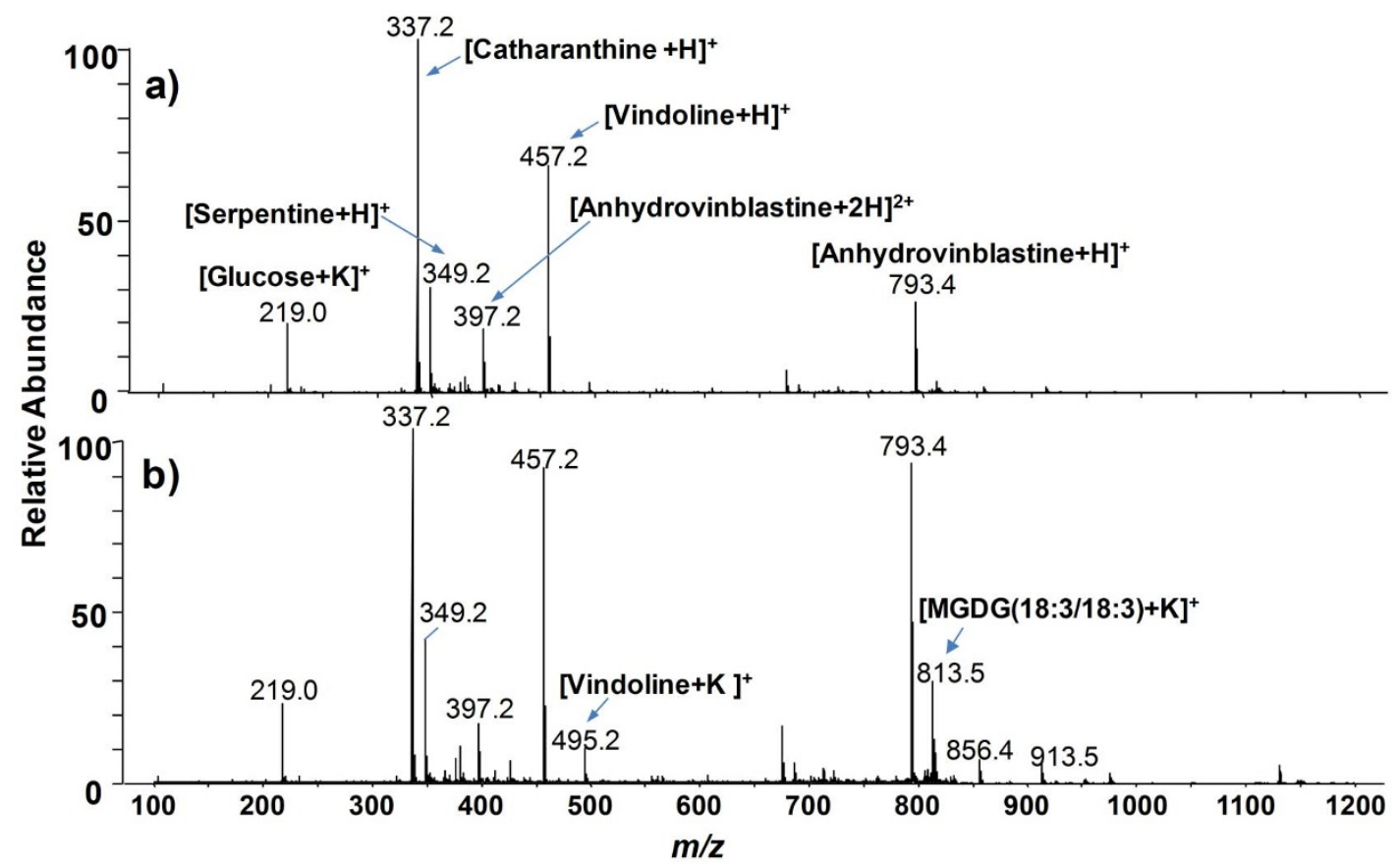

Figure $4 \mid$ Field-induced DI spectra obtained for a leaf of a living $C$. rosues (a) before and (b) after heating. Methanol (2 $\mu \mathrm{L})$ was used as the added solvent. 
(Vcap), that is the voltage applied to the MS inlet, was set at $-5 \mathrm{kV}$ for positive ion mode. Drying gas was used with a temperature of $100^{\circ} \mathrm{C}$ and a flow rate of $4.0 \mathrm{~L} / \mathrm{min}$. ESI-MS and MS/MS experiments were performed on a QToF II mass spectrometer (Micromass, UK) for confirmation of peak assignments. Some of the mass spectral results and the experimental details are shown in Fig. S3 and Fig. S5.

1. Roepke, J. et al. Vinca drug components accumulate exclusively in leaf exudates of Madagascar periwinkle. Proc Natl Acad Sci USA 107, 15287-15292 (2010).

2. Kessler, A. \& Baldwin, I. T. Defensive function of herbivore-induced plant volatile emissions in nature. Science 291, 2141-2144 (2001).

3. Glinski, M. \& Weckwerth, W. The role of mass spectrometry in plant systems biology. Mass Spectrom Rev 25, 173-214 (2006).

4. Chughtai, K. \& Heeren, R. M. A. Mass spectrometric imaging for biomedical tissue analysis. Chem Rev 110, 3237-3277 (2010).

5. Dunn, W. B., Broadhurst, D. I., Atherton, H. J., Goodacre, R. \& Griffin, J. L. Systems level studies of mammalian metabolomes: the roles of mass spectrometry and nuclear magnetic resonance spectroscopy. Chem Soc Rev 40, 387-426 (2011).

6. Cooks, R. G., Ouyang, Z., Takats, Z. \& Wiseman, J. M. Ambient mass spectrometry. Science 311, 1566-1570 (2006).

7. Alberici, R. M. et al. Ambient mass spectrometry: bringing MS into the "real world". Anal Bioanal Chem 398, 265-294 (2010).

8. Huang, M. Z., Yuan, C. H., Cheng, S. C., Cho, Y. T. \& Shiea, J. Ambient Ionization Mass Spectrometry. Annu Rev Anal Chem 3, 43-65 (2010).

9. Yao, Z. P. Characterization of proteins by ambient mass spectrometry. Mass Spectrom Rev 31, 437-447 (2012).

10. Takats, Z., Wiseman, J. M., Gologan, B. \& Cooks, R. G. Mass spectrometry sampling under ambient conditions with desorption electrospray ionization. Science 306, 471-473 (2004).

11. Cooks, R. G., Talaty, N. \& Takats, Z. Rapid in situ detection of alkaloids in plant tissue under ambient conditions using desorption electrospray ionization. Analyst 130, 1624-1633 (2005)

12. Yew, J. Y., Cody, R. B. \& Kravitz, E. A. Cuticular hydrocarbon analysis of an awake behaving fly using direct analysis in real-time time-of-flight mass spectrometry. Proc Natl Acad Sci USA 105, 7135-7140 (2008).

13. Chen, H., Yang, S., Wortmann, A. \& Zenobi, R. Neutral desorption sampling of living objects for rapid analysis by extractive electrospray ionization mass spectrometry. Angew Chem Int Ed 46, 7591-7594 (2007).

14. Chen, H. W. \& Zenobi, R. Neutral desorption sampling of biological surfaces for rapid chemical characterization by extractive electrospray ionization mass spectrometry. Nat Protocols 3, 1467-1475 (2008).

15. Hu, B., Lai, Y. H., So, P. K., Chen, H. W. \& Yao, Z. P. Direct ionization of biological tissue for mass spectrometric analysis. Analyst 137, 3613-3619 (2012).

16. Liu, J. J., Wang, H., Cooks, R. G. \& Ouyang, Z. Leaf Spray: Direct Chemical Analysis of Plant Material and Living Plants by Mass Spectrometry. Anal Chem 83, 7608-7613 (2011).

17. Chan, S. L. F., Wong, M. Y. M., Tang, H. W., Che, C. M. \& Ng, K. M. Tissue-spray ionization mass spectrometry for raw herb analysis. Rapid Commun Mass Spectrom 25, 2837-2843 (2011)

18. RomiLebrun, R. et al. Characterization of four toxins from Buthus martensi scorpion venom, which act on apamin-sensitive $\mathrm{Ca} 2+$-activated $\mathrm{K}+$ channels. Eur J Biochem 245, 457-464 (1997).

19. Hassani, O. et al. Role of lysine and tryptophan residues in the biological activity of toxin VII (Ts gamma) from the scorpion Tityus serrulatus. Eur J Biochem 260, 76-86 (1999).
20. Ali, S. A., Stoeva, S., Grossmann, J. G., Abbasi, A. \& Voelter, W. Purification, characterization, and primary structure of four depressant insect-selective neurotoxin analogs from scorpion (Buthus sindicus) venom. Arch Biochem Biophys 391, 197-206 (2001).

21. Weisel-Eichler, A. \& Libersat, F. Venom effects on monoaminergic systems. J Comp Physiol A -Neuroethol Sens Neural Behav Physiol 190, 683-690 (2004).

22. Zeng, X. C., Luo, F. \& Li, W. X. Molecular dissection of venom from Chinese scorpion Mesobuthus martensii: Identification and characterization of four novel disulfide-bridged venom peptides. Peptides 27, 1745-1754 (2006).

23. Marie, Z. A. \& Ibrahim, S. A. Lipid-content of scorpion (Leiurus-Quinquestriatus, $\mathrm{H}$ and $\mathrm{E}$ ) venom. Toxicon 14, 93-966 (1976).

24. Hadley, N. F. \& Jackson, L. L. Chemical composition of epicuticular lipids of scorpion, Paruroctonus-Mesaensis. Insect Biochem 7, 85-89 (1977).

25. Delfino, G., Brizzi, R. \& Feri, L. Chemical skin defence in Bufo bufo: An ultrastructural study during ontogenesis. Zool Anz 234, 101-111 (1995).

26. Peng, Y. E. et al. In vivo nanoelectrospray for the localization of bioactive molecules in plants by mass spectrometry. Anal Chem 84, 3058-3062 (2012).

27. Tian, H. Y. et al. Bufogargarizins A and B: two novel 19-norbufadienolides with unprecedented skeletons from the venom of Bufo bufo gargarizans. Chem-Eur J 16, 10989-10993 (2010).

28. Gao, H. M., Popescu, R., Kopp, B. \& Wang, Z. M. Bufadienolides and their antitumor activity. Nat Prod Rep 28, 953-969 (2011).

29. Fukuyama, T. et al. Stereocontrolled total synthesis of (+)-vinblastine. J Am Chem Soc 124, 2137-2139 (2002).

30. Farooq, M., Wahid, A., Kobayashi, N., Fujita, D. \& Basra, S. M. A. Plant drought stress: effects, mechanisms and management. Agron Sustain Dev 29, 185-212 (2009).

31. Ibrahim, A. et al. The alphabet of galactolipids in Arabidopsis thaliana. Plant Sci 2 , 1-24 (2011).

\section{Acknowledgements}

This work was supported by The Hong Kong Polytechnic University (Grants A-PD1C, A-PK82, A-PL48 and G-YM52) and Shenzhen Key Laboratory Advancement Program (Project ZDSY20120618173912712).

\section{Author contributions}

B.H., L.W., W.C.Y. and Z.P.Y. designed the research. B.H. and L.W. performed the experiments and analysed the data. Z.P.Y. wrote the manuscript based on discussions with and contributions from all of the authors.

\section{Additional information}

Supplementary information accompanies this paper at http://www.nature.com/ scientificreports

Competing financial interests: The authors declare no competing financial interests.

How to cite this article: Hu, B., Wang, L., Ye, W.C. \& Yao, Z.P. In Vivo and Real-time Monitoring of Secondary Metabolites of Living Organisms by Mass Spectrometry. Sci. Rep. 3, 2104; DOI:10.1038/srep02104 (2013).

This work is licensed under a Creative Commons AttributionNonCommercial-ShareAlike 3.0 Unported license. To view a copy of this license, visit http://creativecommons.org/licenses/by-nc-sa/3.0 